\title{
Applications of Recombinant Monoclonal Antibodies against Filarial Antigen Proteins
}

\author{
Anizah Rahumatullah, Muhammad Hafiznur Yunus, Gee Jun Tye, and Rahmah Noordin* \\ Institute for Research in Molecular Medicine (INFORMM), Universiti Sains Malaysia, Pulau Pinang, Malaysia
}

\begin{abstract}
This study investigated the applications of recombinant monoclonal antibodies (rmAbs) produced against two recombinant filarial proteins of diagnostic value. Ab5B and $A b 3 A$ were produced against recombinant $B m S X P$, and Ab4 and Ab4-fragment crystallizable (Fc) against recombinant BmR1. Ab5B and Ab4-Fc were found to be useful as quality control (QC) reagents for two commercial rapid test kits, such as Brugia Rapid ${ }^{\mathrm{TM}}$ and BLF Rapid ${ }^{\circledR}$ (Reszon Diagnostics International Sdn. Bhd., 47600 Subang Jaya, Selangor, Malaysia), respectively. The two rmAbs reacted positively with the corresponding recombinant proteins lined on the nitrocellulose strips of the cassette tests, thus may replace or reduce the need for patient serum samples as positive controls for QC of the commercial kits. They were also successfully conjugated to gold nanoparticles and reacted positively with the test lines containing the corresponding recombinant proteins when directly applied to the cassette tests. The gold-conjugated reagents can be used to confirm the antigenicity of test lines after the storage of the rapid tests for a prolonged period or under unfavorable conditions. Furthermore, Ab5B and Ab3A were shown to be able to capture the target recombinant proteins through immunoaffinity purification, enabling their use for applications that need very highly purified proteins. In conclusion, this study demonstrated several potential uses of rmAb proteins produced against recombinant filarial proteins.
\end{abstract}

\section{INTRODUCTION}

Lymphatic filariasis (LF) is a mosquito-borne parasitic disease that is estimated to affect 67.88 million people in 73 countries, causing morbidity and disability. ${ }^{1}$ Brugia malayi and Brugia timori cause brugian filariasis, whereas Wuchereria bancrofti causes bancroftian filariasis. The WHO has initiated a global program to eliminate LF as a public health problem (GPELF), and the target year to achieve this objective is $2020{ }^{2}$

Brugia Rapid is a lateral flow rapid test that is being used in the transmission assessment surveys of the elimination program in brugian filariasis-endemic areas. It uses BmR1 recombinant protein as the test line. Another highly sensitive and specific rapid test reported for detection of bancroftian filariasis is the BLF Rapid test comprising BmSXP recombinant protein as the test line. ${ }^{3}$ Reszon Diagnostics International Sdn. Bhd. (Selangor, Malaysia) manufactures both rapid tests. Although many endemic countries would have eliminated LF as a public health problem by year 2020, surveillance for transmission of the infection must still be performed for several years after that to ensure no re-emergence of the disease. The surveillance is especially crucial for brugian filariasis because $B$. malayi is a zoonotic parasite in some settings. Thus, the commercial production of the two kits needs to continue beyond the year 2020 .

As part of the quality control (QC) process during manufacturing of the rapid tests, positive and negative serum samples are used to determine the diagnostic sensitivity and specificity. However, it is getting more challenging to obtain serum samples from LF patients to confirm reactivity/sensitivity of the tests. This difficulty is because of significant reduction in LF cases due to success of the GPELF. ${ }^{2,4}$ Thus, there is a need to address this challenge, which may affect the sustainability of the test production. One way is by using a recombinant monoclonal antibody (rmAb) protein as a $\mathrm{QC}$ reagent.

\footnotetext{
*Address correspondence to Rahmah Noordin, Institute for Research in Molecular Medicine (INFORMM), Universiri Sains Malaysia (USM), 11800 Gelugor, Penang, Malaysia. E-mails: rahmah8485@gmail.com or rahmah@usm.my
}

Previously, our group had reported the isolation of highly specific and sensitive novel rmAb proteins using phage display technology. ${ }^{5,6}$ The present study aimed to develop potential applications of the rmAb proteins. Two of the rmAb proteins were tested as direct $\mathrm{QC}$ reagents by replacing the filarial patient serum samples when testing the diagnostic sensitivity of the kits. Subsequently, the rmAb proteins were conjugated with gold nanoparticles and tested with the rapid tests to confirm their binding with the corresponding recombinant proteins on the test lines. Another application of the rmAb proteins was their use in immunoaffinity purification to produce highly purified recombinant proteins.

\section{MATERIALS AND METHODS}

Production, purification, and verification of $\mathbf{r m A b}$ proteins. The monoclonal antibodies against BmSXP and $\mathrm{BmR} 1$ proteins were isolated using phage display technology, as previously described. ${ }^{5}$ The monoclonal antibody protein expression and purification were performed as previously described. ${ }^{6}$ The preparation of Ab4-Fc is described elsewhere (article submitted for publication). In brief, Ab4Fc plasmid was first constructed, then transfected into HEK293F cell lines (Thermo Fisher Scientific, Waltham, MA). The cells were harvested, and the supernatant containing the protein was purified using Protein A affinity chromatography.

Colloidal gold conjugation to rmAb protein. Conjugation of the antibody to colloidal gold nanoparticles (40 nm) was performed by the nanobiotechnology laboratory of our institute using a previously described method. ${ }^{7}$

Evaluation of rmAb and gold-conjugated rmAb proteins as the $\mathbf{Q C}$ reagent. An initial evaluation of the rmAb proteins (Ab5B, Ab4, and Ab4-Fc) as the $\mathrm{QC}$ reagent was performed using the commercially available Brugia Rapid and BLF Rapid according to the manufacturer's instructions. As positive controls that the test was working well, a serum sample from a W. bancrofti patient was tested with BLF Rapid, and a serum sample from a B. malayi patient was tested with Brugia Rapid. On the other hand, the evaluation of the gold-conjugated rmAb proteins (Ab5B, Ab4, and Ab4-Fc) was performed using 
a lateral flow dipstick lined separately with $1 \mathrm{mg} / \mathrm{mL}$ of BmSXP or BmR1 recombinant antigens. The lateral flow dipstick was prepared and tested, as previously reported. ${ }^{8}$ The volume of the gold-conjugated rmAb proteins (optical density 10) used was $20 \mu \mathrm{L}$. For both tests, the appearance of a red-purplish colored line at the test line signified a positive result, whereas no line observed signified a negative result. The testing took approximately 15 minutes to complete; and if no line was visible after 30 minutes, the test result was confirmed as negative.

Immunoaffinity purification. The immunoaffinity purification was performed as previously described. ${ }^{6} \mathrm{Ab} 5 \mathrm{~B}$ and $\mathrm{Ab} 3 \mathrm{~A}$ were coupled to cyanogen bromide-activated sepharose resin based on the previously published protocol. ${ }^{9}$ The coupling efficiency was calculated by dividing the supernatant of antibody protein before and after coupling and analyzed using sodium dodecyl sulphate-polyacrylamide gel electrophoresis (SDS-PAGE). Then, the rmAb protein-coupled resin was packed into individual and mixture columns. The binding efficiency of the columns were evaluated using His-tag affinity-purified protein and analyzed using SDS PAGE.

\section{RESULTS}

The rmAb proteins were successfully expressed and purified and were observed on SDS-PAGE at the expected molecular masses of approximately $35 \mathrm{kDa}$ for Ab5B, Ab3A, and $\mathrm{Ab} 4$ and $70 \mathrm{kDa}$ for Ab4-Fc. Western blot using the respective recombinant antibodies showed bands at the same approximate molecular masses.

Then, the rmAb proteins were directly tested as QC reagents (Figure 1). Brugia Rapid and BLF Rapid contain recombinant $B m R 1$ and $B m S X P$, respectively, lined at position $\mathrm{C}$ (test line). The Ab5B protein showed a positive reaction with BLF Rapid and a negative reaction with Brugia Rapid, thus showing the specific binding of Ab5B to BmSXP recombinant protein. Meanwhile, Ab4-Fc showed positive reaction with Brugia Rapid and negative reaction with BLF
Rapid, thus showing the specific binding of Ab4-Fc to BmR1 recombinant protein. However, Ab4 did not show reactivity with Brugia Rapid. When filarial patients' serum samples (positive controls) were tested with the diagnostic kits, they showed the expected positive reaction. Subsequently, the rmAb proteins were gold-conjugated, and binding with the lined BmSXP or BmR1 on the cassette tests was determined (Figure 2). Ab5B and Ab4-Fc showed positive reactions against BmSXP and BmR1, respectively. Meanwhile, Ab4 was not reactive with the BmR1 line on Brugia Rapid.

The immunoaffinity purification was performed using Ab5B and $A b 3 A$. A similar assay using Ab4 had previously been published. ${ }^{6}$ Both rmAb proteins (Ab5B and Ab3A) were successfully coupled and packaged to produce the affinity columns. The coupling efficiency was satisfactory, at $80 \%$ for Ab5B and $69 \%$ for Ab3A. Single and mixture columns were evaluated using Histag affinity-purified BmSXP recombinant protein. Both columns showed functional specificity in capturing the target antigen; however, the mixture rmAb protein column showed better target antigen recovery than the single rmAb protein column (Figure 3 ).

\section{DISCUSSION}

In the present study, three of the four rmAb proteins (Ab4, $A b 5 B$, and $A b 3 A$ ) were in the form of single chain fragment variables (scFv). Meanwhile, Ab4-Fc is Ab4 that has been fused with the Fc region of IgG4. The rmAb proteins were tested for use as $\mathrm{QC}$ reagents and in immunoaffinity assay.

$\mathrm{Ab5B}$, which is a BmSXP-specific antibody protein, showed a positive reaction with BLF Rapid containing BmSXP as the test line. On the other hand, Ab4, which is a BmR1-specific antibody protein, did not show reactivity with Brugia Rapid (lined with BmR1), whereas Ab4-Fc showed positive reaction. The difference in the reactivity of Ab4 and Ab4-Fc when tested with Brugia Rapid was notable. From our previous work, Ab4 showed high specificity and sensitivity to BmR1 when tested in an ELISA. ${ }^{6}$ The different antibody formats between the two
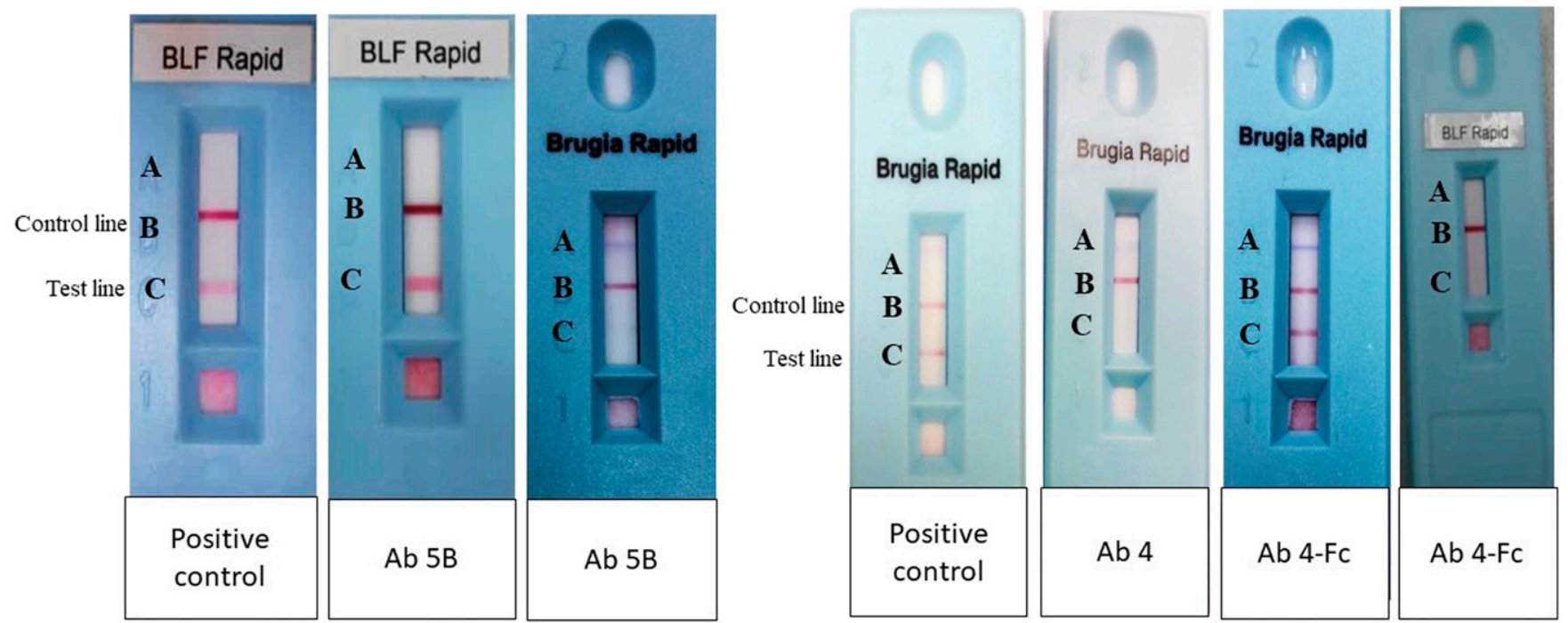

FIGURE 1. Application of recombinant monoclonal antibody proteins as a direct quality control reagent. The left group of tests shows that Ab5B protein gave positive reaction with the BLF Rapid test and negative reaction with Brugia Rapid at test line $C$. Positive control comprised the serum sample from a Wuchereria bancrofti patient tested with BLF Rapid. The right hand group of tests shows that Ab4 did not react with Brugia Rapid; however, Ab4-Fc gave positive reaction with Brugia Rapid (at test line C) and negative reaction with BLF Rapid. Positive control comprised the serum sample from a Brugia malayi patient tested with Brugia Rapid. This figure appears in color at www.ajtmh.org. 


\section{Ab 5B: anti-BmSXP}

BmSXP dipstick B $m$ R1 dipstick

Conjugate: $\mathrm{Ab} 5 \mathrm{~B}$ Conjugate: $\mathrm{Ab} 5 \mathrm{~B}$
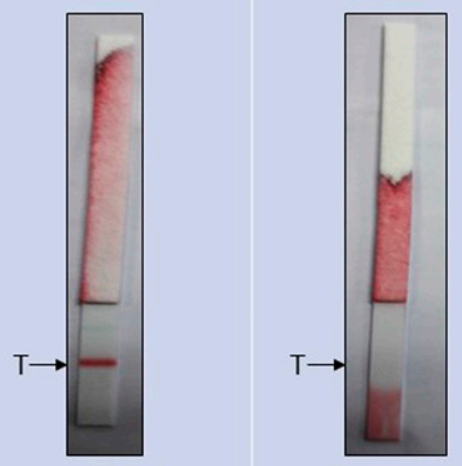

\section{Ab 4-Fc: anti-B $m$ R1}

BmSXP dipstick

Conjugate: $\mathrm{Ab} 4-\mathrm{Fc}$

$\mathrm{B} m \mathrm{R} 1$ dipstick

Conjugate: Ab4-Fc

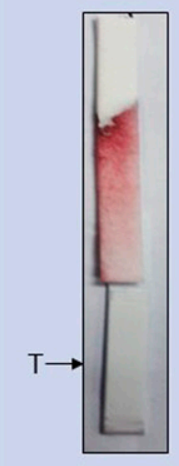

\section{Ab 4: anti-BmR1}

B $m$ R1 dipstick

Conjugate: $\mathrm{Ab} 4$

BmSXP dipstick

Conjugate: $\mathrm{Ab} 4$
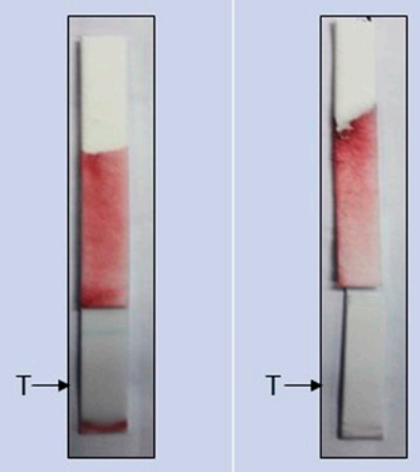

FIGURE 2. Application of recombinant monoclonal antibody proteins as gold-conjugated antibodies to check antigenicity of the lined proteins. Gold-conjugated Ab5B protein showed positive reaction with BmSXP-lined dipstick and no reaction on BmR1-lined dipstick. Gold-conjugated Ab4-Fc protein showed positive reaction on BmR1-lined dipstick and no reaction on BmSXP-lined dipstick. Gold-conjugated Ab4 showed no reaction on both dipsticks. T indicates where the recombinant antibody protein was lined (test line). This figure appears in color at www.ajtmh.org.

rmAb proteins (i.e., scFV versus scFv-Fc) may account for this difference. Theoretically, the addition of Fc increases the protein stability, and binding affinity decreases the propensity of protein aggregation and recovers the Fc function. ${ }^{10,11}$ Certainly, in this study, the addition of the Fc region seemed to improve the diagnostic sensitivity of Ab4 when tested with Brugia Rapid. However, it is difficult to formulate a specific explanation on why Ab4 did not bind the BmR1 line on the rapid test. Further investigations need to be performed to elucidate the possible reasons, such as by analyzing the antigen and antibody interactions using surface plasmon resonance. This method allows real-time monitoring of antigen-antibody complex interactions by providing the equilibrium dissociation constant $(K D)$ value. Methods such as fluorescence ELISA ${ }^{12}$ and kinetic exclusion ${ }^{13}$ assays are also other technologies that can be used to determine the KD value. Furthermore,
A

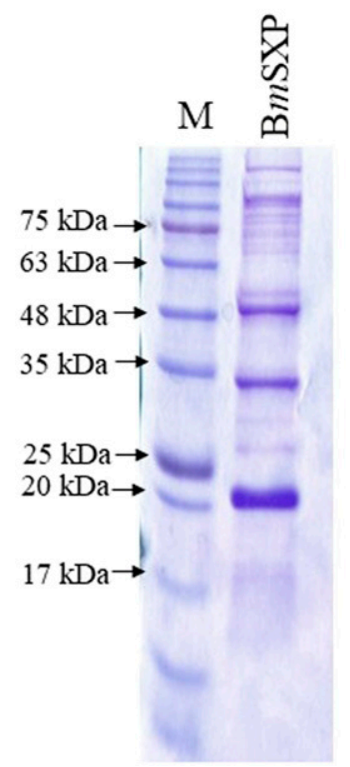

B

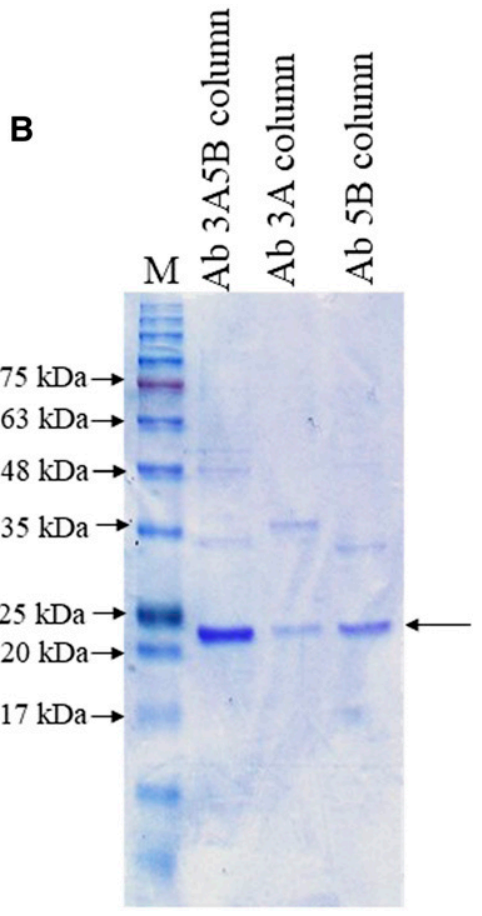

FIGURE 3. SDS PAGE analysis of the outcome of the immunoaffinity purification, and evaluation of binding of Ab5B and Ab3A using purified BmSXP antigen. (A) shows a prominent band of purified BmSXP band at $\sim 22 \mathrm{kDa}$, the additional bands above it are probably aggregated BmSXP. (B) shows that both antibody columns (Ab3A and Ab5B) demonstrated good specificity in capturing the target BmSXP antigen; however, the mixture protein column (Ab3A5B) showed better target antigen recovery than the single protein column. This figure appears in color at www.ajtmh.org. 
structural bioinformatic studies, such as molecular docking and antibody structure modeling, may give insights into the biophysical properties of an antibody molecule and the binding epitope involved during antibody-antigen interaction. ${ }^{14,15}$

When gold-conjugated rmAb proteins were tested with the commercial kits, Ab5B and Ab4-Fc showed positive reactions against BLF Rapid and Brugia Rapid, respectively. Rapid tests that have been stored for some time under non-optimal conditions can be tested with the gold-conjugated antibodies to confirm whether the recombinant proteins lined on the nitrocellulose strips still retain their antigenicity.

The strong binding to their targets makes antibodies suited as immobilized ligands. ${ }^{16-18}$ In this regard, the two BmSXPspecific rmAbs ( $5 B$ and $3 A$ ) were used in immunoaffinity purification. The rmAb proteins were able to specifically capture and release the target antigens from a pool of proteins, and the best yield was obtained when the two antibody proteins were combined. The eluted protein can be used for applications that require highly purified proteins such as for crystallography analysis and biosensor development. The immunoaffinity column may also be used to characterize the circulating filarial antigens captured by the recently reported $W$. bancroft $i$ antigen-detection 5B-ELISA. ${ }^{19}$

In summary, this study demonstrated three applications of rmAb proteins. Ab5B and Ab4-Fc successfully showed good performance as direct $Q C$ reagents for Brugia Rapid and BLF Rapid test, respectively. Gold-conjugated Ab5B and Ab4-Fc showed excellent sensitivity toward their respective target antigens and, thus, may be used to check the antigenicity of test lines after being stored for prolonged periods or under unfavorable conditions. The test users may acquire the rmAb reagents for batch-testing of the rapid tests to confirm that they are working before using them in the field. Also, the immunoaffinity purification showed that $A b 5 B$ and $A b 3 A$ were able to capture BmSXP recombinant protein from a pool of proteins.

Received October 20, 2019. Accepted for publication November 25, 2019.

Published online January 13, 2020.

Acknowledgment: We acknowledge Universiti Sains Malaysia for funding the postdoctoral position of A. R.

Financial support: This study was supported by a grant from the Malaysian Ministry of Education through the Higher Institution Center of Excellence (HICoE) Grant (Grant No. 311/CIPPM/4401005).

Disclosure: R. N., A. R., and M. H. Y. reports a sale of Ab5B (against recombinant BmSXP protein) was made via the university commercial arm to the Reszon Diagnostics International. Sdn. Bhd. received a small fraction of the sale value.

Authors' addresses: Anizah Rahumatullah, Muhammad Hafiznur Yunus, Gee Jun Tye, and Rahmah Noordin, Institute for Research in Molecular Medicine (INFORMM), Universiti Sains Malaysia, Pulau Pinang, Malaysia, E-mails: anirah82@gmail.com, mhn1016@yahoo.com, geejun@usm.my, and rahmah8485@gmail.com.

\section{REFERENCES}

1. Ramaiah KD, Ottesen EA, 2014. Progress and impact of 13 years of the global programme to eliminate lymphatic filariasis on reducing the burden of filarial disease. PLoS Negl Trop Dis 8: e3319.

2. Ichimori K, King JD, Engels D, Yajima A, Mikhailov A, Lammie P, Ottesen EA, 2014. Global programme to eliminate lymphatic filariasis: the processes underlying programme success. PLOS Negl Trop Dis 8: e3328.

3. Noordin R, Yunus MH, Robinson K, Won KY, Babu S, Fischer PU, Hisam S, Mahmud R, 2018. Laboratory evaluation of a rapid IgG4 antibody test (BLF rapid) for bancroftian filariasis. Am J Trop Med Hyg 99: 1587-1590.

4. Turner HC, Bettis AA, Chu BK, McFarland DA, Hooper PJ, Mante SD, Fitzpatrick C, Bradley MH, 2017. Investment success in public health: an analysis of the cost-effectiveness and costbenefit of the global programme to eliminate lymphatic filariasis. Clin Infect Dis 64: 728-735.

5. Rahumatullah A, Ahmad A, Noordin R, Lim TS, 2015. Delineation of BmSXP antibody V-gene usage from a lymphatic filariasis based immune scFv antibody library. Mol Immunol 67: 512523.

6. Rahumatullah A, Abdul Karim IZ, Noordin R, Lim TS, 2017. Antibody-based protective immunity against helminth infections: antibody phage display derived antibodies against BmR1 antigen. Int J Mol Sci 18: E2376.

7. Makhsin SR, Razak KA, Noordin R, Zakaria ND, Chun TS, 2012. The effects of size and synthesis methods of gold nanoparticleconjugated MalphaHlgG4 for use in an immunochromatographic strip test to detect brugian filariasis. Nanotechnology 23: 495-719.

8. Yunus MH, Tan Farrizam SN, Abdul Karim IZ, Noordin R, 2018. A lateral flow rapid test for human toxocariasis developed using three toxocara canis recombinant antigens. Am J Trop Med Hyg 98: 32-38.

9. Kavran JM, Leahy DJ, 2014. Coupling antibody to cyanogen bromide-activated sepharose. Methods Enzymol 541: 27-34.

10. Nian S, Wu T, Ye Y, Wang X, Xu W, Yuan Q, 2016. Development and identification of fully human scFv-Fcs against Staphylococcus aureus. BMC Immunol 17: 8 .

11. Jorgensen ML, Friis NA, Just J, Madsen P, Petersen SV, Kristensen $P, 2014$. Expression of single-chain variable fragments fused with the Fc-region of rabbit IgG in Leishmania tarentolae. Microb Cell Fact 13: 9.

12. High K, Meng Y, Washabaugh MW, Zhao Q, 2005. Determination of picomolar equilibrium dissociation constants in solution by enzyme-linked immunosorbent assay with fluorescence detection. Anal Biochem 347: 159-161.

13. Bee C, Abdiche YN, Stone DM, Collier S, Lindquist KC, Pinkerton $A C$, Pons J, Rajpal A, 2012. Exploring the dynamic range of the kinetic exclusion assay in characterizing antigen-antibody interactions. PLoS One 7: e36261.

14. Pedotti M, Simonelli L, Livoti E, Varani L, 2011. Computational docking of antibody-antigen complexes, opportunities and pitfalls illustrated by influenza hemagglutinin. Int J Mol Sci 12: 226-251.

15. Raybould MIJ, Marks C, Krawczyk K, Taddese B, Nowak J, Lewis AP, Bujotzek A, Shi J, Deane CM, 2019. Five computational developability guidelines for therapeutic antibody profiling. Proc Natl Acad Sci USA 116: 4025-4030.

16. Hage DS, 1998. Survey of recent advances in analytical applications of immunoaffinity chromatography. J Chromatogr $B$ Biomed Sci Appl 715: 3-28.

17. Calton GJ, 1984. Immunosorbent separations. Methods Enzymol 104: 381-387.

18. Phillips TM, 1989. High-performance immunoaffinity chromatography. Adv Chromatogr 29: 133-173.

19. Rahumatullah A, Lim TS, Yunus MH, Noordin R, 2019. Development of an antigen detection ELISA for bancroftian filariasis using BmSXP-specific recombinant monoclonal antibody. Am J Trop Med Hyg 101: 436-440. 